
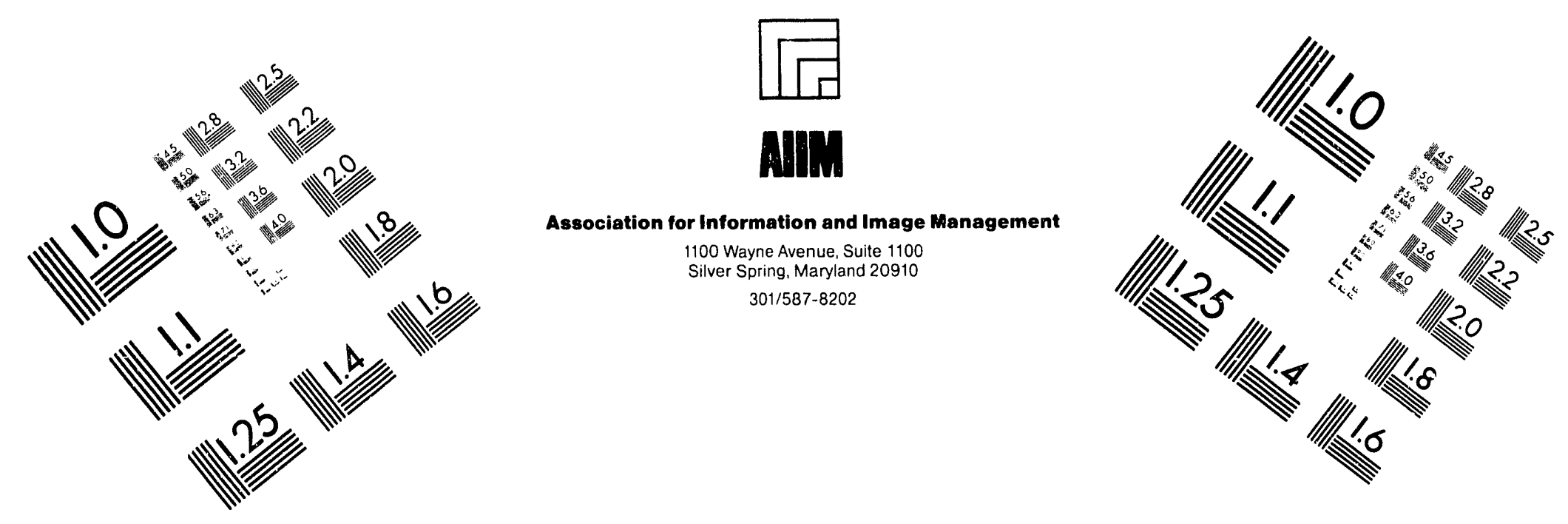

Centimeter

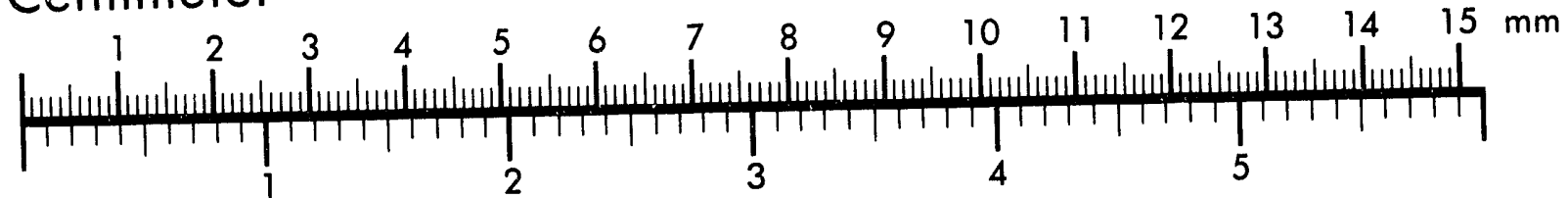
Inches
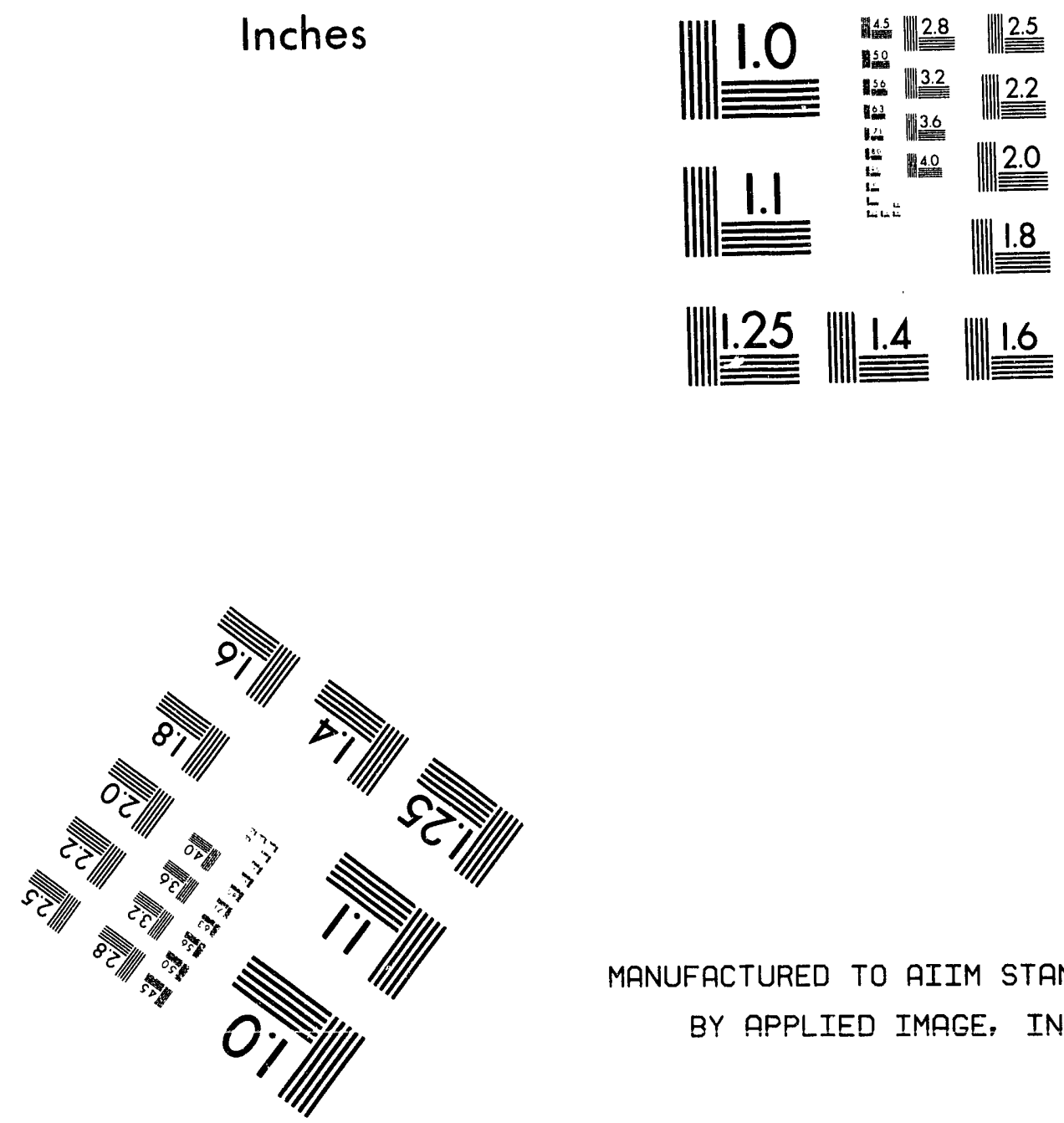

MANUFACTURED TO AIIM STANDARDS

BY APPLIED IMAGE, INC.

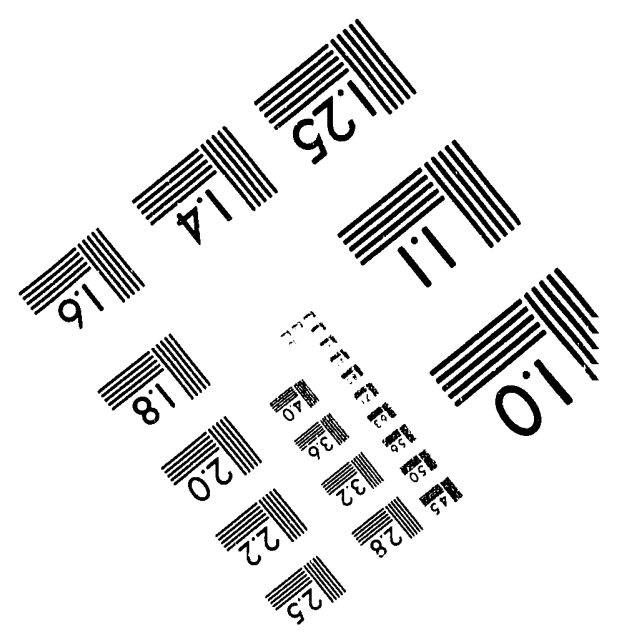



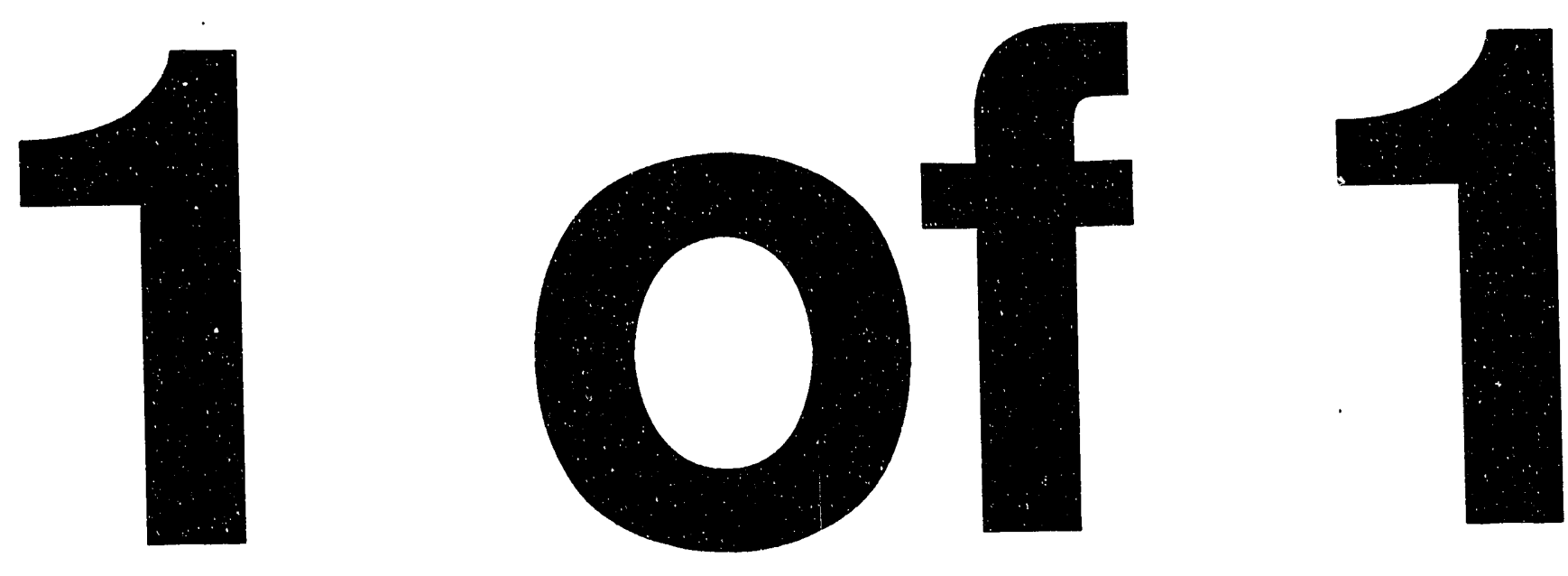
1993 Particle Accelerator Conf., Washington, D.C., Yav 17-20, 1993

\title{
Time Domain Solutions for a Coasting Beam with Impedance Feedback
}

\author{
M. Blaskiewicz \\ AGS Dept. Brookhaven National Lab \\ BNL 911C Upton, NY 11973
}

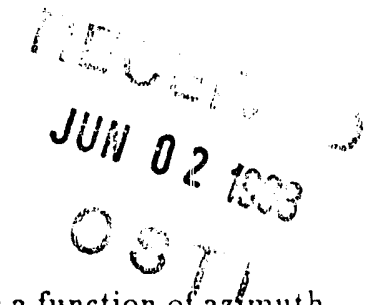

\section{Abstract}

Time domain solutions for a coasting beam interacting with a longitudinal or transverse impedance are presented. The treatment is limited to first order perturbation theory, but it includes Landau damping.

\section{INTRODUCTION}

Analytic treatments of the linearized Vlasov equation are usually posed as cigenvalue problems in the frequency domain $\{1,2,3\}$, while computer codes usually work in the time domain. In this note, a time domain approach to the linearized vlasov equation is introduced. The technique is applied to both longitudinal and transverse coasting beam instabilities. In both cases, the Vlasov equation is reduced to a nne dimensional Volterra equation of the second kind. Analvtic solutions for specialized distributions are presented. The solutions are easily expressed in simple functions, allowing for detailed analytic scrutiny, as well as making them casy to include in computer code. The following two sections focus on the longitudinal case. The transverse case is presented in section IV.

\section{THEORETICAL DEVELOPMENT}

For a coasting beam with self forces due to a longitudinal wake potential, the Vlasov equation reads

$$
\frac{\partial \psi(\theta, \delta, t)}{\partial t}+\omega_{11}(1-\eta \delta) \frac{\partial \psi(\theta, \delta, t)}{\partial \theta}+\dot{\delta} \frac{\partial \psi(\theta, \delta, t)}{\partial \delta}=0 .
$$

In equation (1), $\theta$ is the azimuth around the machine, $\omega_{0}$ is the angular revolution frequency for the ideal particle, $\delta=\angle p / p_{0}$ is the fractional momentum deviation, $\eta$ is the frequency slip factor, and $\psi(\theta, \delta, t) d \theta d \delta$ is the number of particles in $d \theta \times d \delta$ at time $t$.

The effect of the wake potential is contained in $\dot{\delta}$. In the smooth approximation:

$$
\dot{\delta}(\theta, t)=-\frac{q}{2 \pi R p_{i 1}} \int_{-\infty}^{\infty} W(\tau) I(\theta, t-\tau) d \tau
$$

where $q$ is the charge on a single particle, $R$ is the machine radius, $W(\tau)$ is the wake potential as a function of time

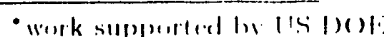

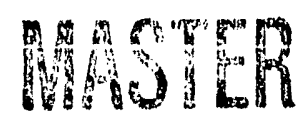

lag, and $I(\theta, t)$ is the beam current as a function of azmuth and time.

As usual, assume that the phase space distribution is given by an unperturbed piece, plus a small perturbation. In the limit of first order perturbation theory, the azimuthal harmonics are orthogonal and may be studied individually:

$$
\psi(\theta, \delta, t)=\psi_{0}(\delta)+\psi_{n}(\delta, t) e^{i n\left(\theta-\omega_{1,} t\right)} .
$$

It is assumed that the wake force due to $\psi_{11}$ vanishes. 'Then. the wake force is soley due to the perturbation current This force is given by

$$
\dot{\delta}=-\frac{q^{2} \omega_{n}}{2 \pi R p_{11}} e^{i n\left(\theta-\omega_{11} t\right)} \int_{-\infty}^{+\infty} d \tau W(\tau) e^{i n \omega_{11} \tau} \rho_{n}(t-\tau)
$$

where,

$$
\rho_{n}(t)=\int_{-\infty}^{+\infty} \psi_{n}(\delta, t) d \delta
$$

The $\tau$ dependence of $\rho_{n}(t-\tau)$ in the wake force is ignored. This is the only approximation to first order perturbation theory. It assumes that the wake potential decays before $\psi_{n}$ changes appreciably.

Expressing equation (4) in terms of the longitudinal impedance $Z$, the first order solution of equation (1) satisfies

$$
\begin{aligned}
\frac{\partial \psi_{n}(\delta, t)}{\partial t} & =i n \omega_{0} \eta \delta \psi_{n}(\delta, t)+R(\delta) \rho_{n}(t) \\
R(\delta) & =\frac{q^{2} \omega_{0}}{2 \pi R p_{0}} \frac{d \psi_{0}(\delta)}{d \delta} Z\left(n \omega_{0}\right) .
\end{aligned}
$$

Equation (6) is easily solved using $\exp \left(-i n \omega_{0} \eta \delta t\right)$ as an integrating factor,

$$
\begin{aligned}
\psi_{n}(\delta, t) & =\psi_{n}(\delta, 0) e^{i n \omega_{0} \eta \delta t} \\
& +R(\delta) \int_{0}^{l} \rho_{n}(s) e^{i n \omega_{0} \eta \delta(t-s)} d s
\end{aligned}
$$

Before proceeding with the solution, introduce the total number of particles in the ring $N_{t}$ and the characteristic 
half width of the momentum distribution $\sigma$ via

$$
\psi_{11}(\delta)=\frac{N_{1}}{2 \pi \sigma} f(\delta / \sigma)
$$

Integrating both sides of equation (8) over $\delta$ and switching to the dimensionless time variable $\tau=|\eta| n \sigma \omega_{0} t$ results in

$$
\begin{aligned}
\rho_{n}(\tau) & =S(\tau)-i \frac{Z\left(n \omega_{1}\right)}{Z_{\imath} r i t} \int_{0}^{r} \rho_{n}(s) G(\tau-s) d s \\
G(\tau) & =\tau \int_{-\gamma}^{x} f(x) e^{i \operatorname{sgn}(\eta) \tau x} d x \\
Z_{r+1 l} & =n \frac{4 \pi^{2} R p_{1,} \sigma^{2} \eta}{q^{2} N_{l}}
\end{aligned}
$$

In equation (10), $S(\tau)$ is the source function and is given by the integral, over $\delta$, of the first term on the right of equation (8). For reasonable distributions, $G(\tau)$ is a smooth function that viries over length scales of order unity, and has a maximum value of order unity. For the correct choice of $\sigma,|Z, \ldots|$ is the kiril-schnell limiting impedance [1]. The actual stability of the system can be strongly affected by the phase of the impedance as well [3].

\section{EXACT S()IUTION}

An exact solution to equation (10) may be obtained if the unperturbed distribution is a Lorentzian,

$$
f(x)=\frac{1}{\pi\left(1+x^{2}\right)}
$$

where $x=\delta / \sigma$. The response function is

$$
G(\tau)=\tau e^{-|\tau|}
$$

Since $\tau \geq 0$ in our problem, the absolute value sign is not needed. To solve equation (10), proceed by defining the auxiliary variables

$$
\begin{aligned}
\alpha(\tau) & =\int_{0}^{\tau} e^{s} \rho_{n}(s) d s, \\
\beta(\tau) & =\int_{0}^{\tau} s e^{s} \rho_{n}(s) d s, \\
\rho_{n}(\tau) & =e^{-\tau} \dot{\alpha}, \\
\dot{\beta} & =\tau \dot{\alpha},
\end{aligned}
$$

where dot denotes differentiation with respect to $r$, and the second two lines follow from the first two.

Plugging in to equation (10) with equations (14) and (15) gives

$$
\dot{\alpha}=S e^{\tau}+\lambda^{2}(\tau \alpha-\beta)
$$

The effect of the impedance is fully contained in the entlstant

$$
\lambda^{2}=-i \frac{Z\left(n \omega_{11}\right)}{Z_{\text {trit }}} .
$$

Differentiating equation (16) with respect to $\tau$ and using the last line of (15) gives

$$
\ddot{\alpha}-\lambda^{2} \alpha=\frac{d}{d \tau}\left[S(\tau) e^{\tau}\right] .
$$

The boundary conditions at $\tau=0$ are $\alpha=0$, and $\dot{\alpha}=S(0)$. For an initial perturbation that is a Lorentzian,

$$
S(\tau)=S_{0} e^{-\epsilon \tau},
$$

where $\epsilon=\tilde{\sigma}-i z_{0}$. The width of the perturbation in $\varepsilon$ is $\tau \bar{\sigma}$. and the perturbation is centered at $\delta=\operatorname{sgn}(\eta) \sigma z_{11}$. Since the right hand side of equation (18) is an exponential, a - -) is readily obtained. I consider the non-degenerate case. so $\alpha$ is a sum of exponential functions. Using the third line of (15) gives $\rho_{n}(\tau)$,

$$
\begin{aligned}
\rho_{n}(\tau) & =\frac{S_{n}}{(1-\epsilon)^{2}-\lambda^{2}}\left\{(1-\epsilon)^{2} e^{-\epsilon \tau}\right. \\
& \left.-\lambda e^{-\tau}[\lambda \cosh (\lambda \tau)+(1-\epsilon) \sinh (\lambda \tau)\}\right\}(.2111
\end{aligned}
$$

The motion is stable if and only if $|\Re(\lambda)|:=1$. (hanging the momentum variable to $z=\operatorname{sgn}(\eta) \delta / \sigma$, and evaluating equation (8) yields,

$$
\begin{aligned}
\psi_{n}(z, \tau) & =\frac{S_{11} e^{i z \tau}}{\pi}\left\{\frac{\tilde{\sigma}}{\tilde{\sigma}^{2}+\left(z-z_{11}\right)^{2}}\right. \\
& \left.-i \lambda^{2} \frac{2 z}{\left(1+z^{2}\right)^{2}} \frac{F}{\left[(1-\epsilon)^{2}-\lambda^{2}\right]}\right\}
\end{aligned}
$$

where

$$
\begin{aligned}
F & =\frac{(1-\epsilon)^{2}}{(\epsilon+i z)}\left[1-e^{-(\epsilon+i z) \tau}\right] \\
& -\frac{\lambda}{2} \frac{(1+\lambda-\epsilon)}{(1-\lambda+i z)}\left[1-e^{-(1-\lambda+i z) \tau]}\right. \\
& +\frac{\lambda}{2} \frac{(1-\lambda-\epsilon)}{(1+\lambda+i z)}\left[1-e^{-(1+\lambda+i z) \tau]}\right.
\end{aligned}
$$

As required, equations (20) and (21) are invariant under the substitition $\lambda \rightarrow-\lambda$.

If the system is unstable, the distribution at large $\tau$ is given by

$$
\psi_{n}(z, \tau) \approx \frac{C z e^{-(1-\lambda) \tau}}{(1-\lambda+i z)\left(1+z^{2}\right)^{2}}
$$

where $C$ is a constant that depends on the initial conditions and I have taken $\Re(\lambda)>1$. Equation (23) is the unstable eigenvector one gets from a frequency domain treatment as may be verified by substitution into (6) with the appropriate change of variables. As one expects, the stability of the system is determined by the eigenvectors. 


\section{TRANSVERSE CASE}

The solution for a coasting beam subject to a transverse impedance is very analogous to the treatment for the longitudinal impedance, but the treatment is more cumbersome due to the cxtra phase space dimensions.

Let $x$ be the transverse coordinate and $\omega_{x}$ be the average betation frequency. Define $r$ as the amplitude of the betratron oscillation and let $\phi$ be the angle variable. Then

$$
\begin{aligned}
\frac{d \phi}{d t} & =\omega_{x}+\Delta \omega_{r}(r, \delta), \\
\frac{d r}{d t} & =\frac{\sin \phi F_{r}}{\gamma m \omega_{x}}, \\
& \approx \frac{e^{i \phi} F_{x}}{2 i \gamma m \omega_{. r}}
\end{aligned}
$$

where $\Delta \omega_{r}(r, \delta)$ is the amplitude and momentum dependent part of the betation frequency, $F_{r}$ is the transverse force due to the beam offset combined with the transverse impedance, $\gamma$ is the lorentz factor of the beam and $m$ is the particle mass. The approximation (26) is equivalent to assuming that the betatron tune shift is small compared to the betatron tune.

Assume a solution of the form,

$\psi(r, \delta, \theta, \phi, t)=\psi_{11}(r, \delta)+\psi^{\prime} 1(r, \delta, t) e^{i\left[\phi-\omega_{r} t+n\left(\theta-\omega_{11} t\right)\right]}$

By ignoring the variation in $\psi_{1}$ over the time scale for the transverse wake potential to decay, the transverse force is given by,

$$
F_{x}(\theta, t)=\frac{i q \beta Z_{\perp}\left(n \omega_{11}+\omega_{x}\right) D(t)}{2 \pi R} e^{i\left[n\left(\theta-\omega_{0} t\right)-\omega_{x} t\right]}
$$

where $\beta$ is the velocity in units of the speed of light and,

$$
D(t)=\pi q \omega_{11} \int r^{2} d r d \delta \psi_{1}(r, \delta, t)
$$

is the dipole moment of the beam.

The first order Vlasov equation is given by,

$$
\begin{aligned}
\frac{\partial \psi_{1}}{\partial t} & =-i\left[\Delta \omega_{x}(r, \delta)-n \omega_{0} \eta \delta\right] \psi_{1} \\
& +\frac{q \beta Z_{\perp}\left(n \omega_{0}+\omega_{x}\right) D(t)}{4 \pi \gamma m \omega_{x} R} \frac{\partial \psi_{0}}{\partial r} .
\end{aligned}
$$

Using the same techniques as in the longitudinal case, the Vlasov equation can be reduced to an integral equation for the dipole moment,

$$
\begin{aligned}
D(t) & =S_{r}(t)+A \int_{1}^{t} D(s) G(t-s) d s \\
A & =-\frac{q^{2} N_{1} Z_{\perp} \omega_{10} \beta}{8 \pi^{2} R \gamma m \omega_{x}} \\
G(t) & =\int r^{2} d r d \delta \frac{\partial \psi_{0}}{\partial r} e^{i\left(\Delta \omega_{x}(r, \delta)-n \omega_{0} \eta \delta\right) t}
\end{aligned}
$$

where $S_{r}(t)$ is the transverse source function. For the case $\Delta \omega_{x}=\delta Q^{\prime} \omega_{n}$, with Lorentzians for momentum distributions, the equations may be solved.

Define the dimensionless variables $z=\operatorname{sgn}\left(Q^{\prime}-n \cdot \eta\right) \delta / \sigma$ and $\tau=t \sigma \omega_{n}\left|Q^{\prime}-n \eta\right|$. The unperturbed distribution is given by, $\psi_{0}(r, \delta)=T_{11}(r) /\left(1+z^{2}\right)$, and the perturbation at $t=0$ is $\psi_{1}(r, \delta, 0)=C T_{n}^{\prime}(r) \tilde{\sigma} /\left[\tilde{\sigma}^{2}+\left(z-z_{11}\right)^{2}\right]$. The dipole moment of the beam is given by

$$
D(\tau)=S_{0}\left\{\frac{(1-\epsilon) e^{-\epsilon \tau}-\lambda e^{(\lambda-1) \tau}}{1-\lambda-\epsilon}\right\}
$$

where $\lambda=A /\left(\sigma \omega_{n}\left|Q^{\prime}-n \eta\right|\right)$, and $\epsilon=\tilde{\sigma}+i z_{11}$. The perturbed distribution is given by,

$$
\begin{aligned}
\psi_{1}(\delta, r, \tau) & =C \frac{d T_{0}(r)}{d r} e^{-i z \tau}\left[\frac{\tilde{\sigma}}{\tilde{\sigma}^{2}+\left(z \cdots z_{1}\right)^{2}}\right. \\
& \left.+\frac{\lambda F}{\left(1+z^{2}\right)(1-\lambda-\epsilon)}\right] \\
F & =\frac{1-\epsilon}{\epsilon \cdots i z}\left[1-e^{-(\epsilon-i z) \tau}\right] \\
& +\frac{\lambda}{\lambda \cdots 1+i z}\left[1-e^{(\lambda-1+i z) \tau}\right] .
\end{aligned}
$$

\section{CONCLUSIONS}

Two exact solutions to the initial value problem for the Vlasov equation have been presented. Using fairly simple numerical techniques, solutions for a broad range of momentum distributions could be obtained. These solutions might be useful in the development of computer codes, especially when trying to determine the effect of the granularity produced by particle tracking. Additionally, the simplicity of the solutions may allow for a serious study of the analytic properties of Landau damping. Finally, since momentum distributions in real machines can be measured, a relatively simple comparison of theory and experiment. might be possible.

\section{ACKNOWLEDGEMENTS}

My thanks to A. Luccio and W.T. Weng for useful discussions and encouragement.

\section{References}

[1] E. Keil \& W. Schnell, CERN/69-48 (1969).

[2] J. L. Laclare, CERN/85-19 (1985) p 377.

[3] A. G. Ruggiero \& V. G. Vaccaro, CERN ISR-TH/6833 (1968). 

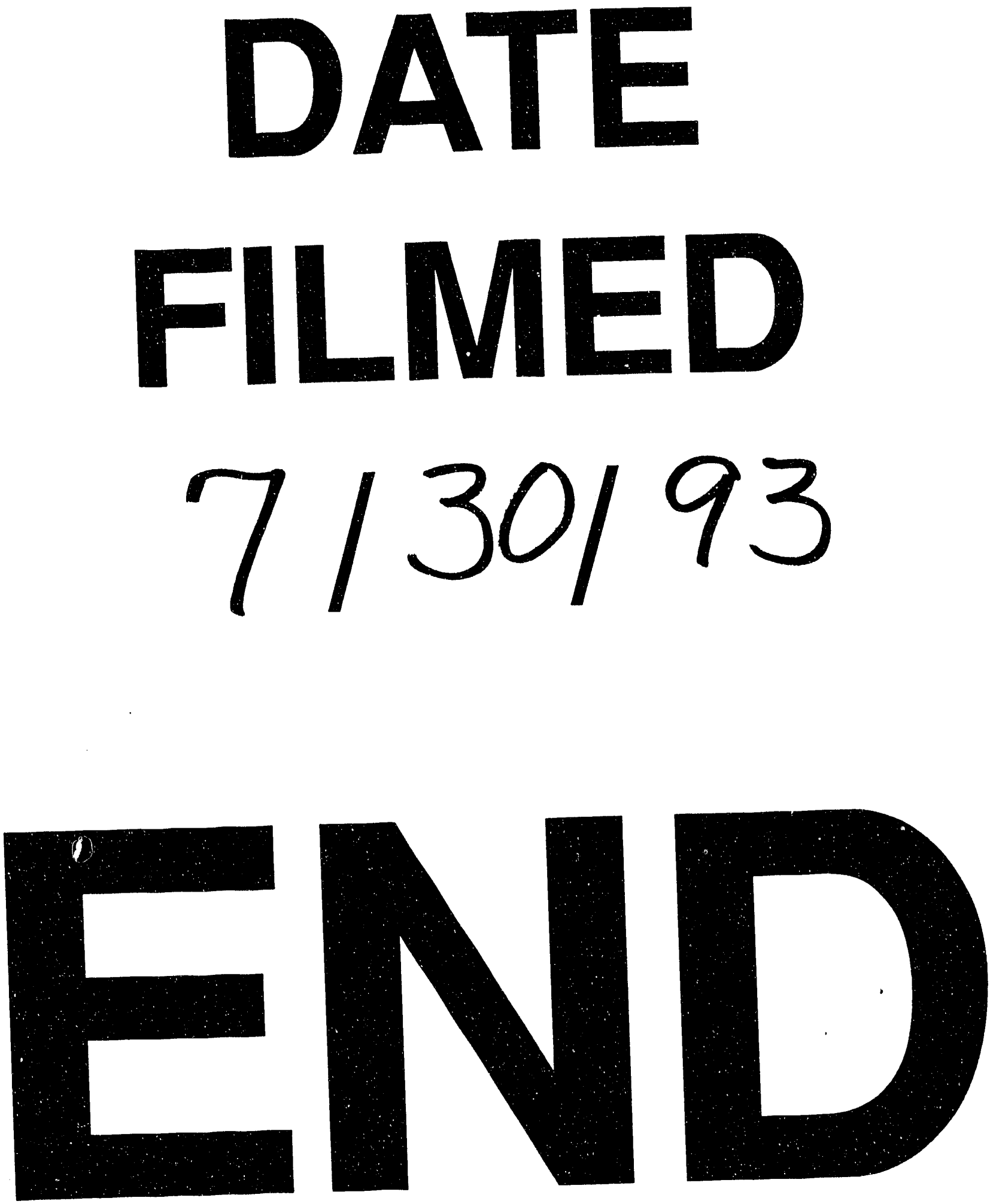
